\title{
Environmental TEM, A CTF Approach for Atmospheric Lattice Imaging
}

\author{
Makoto Suzuki* and Toshie Yaguchi* \\ * Hitachi High-Technologies Corp., 882 Ichige, Hitachinaka, Ibaraki 312-8504, Japan
}

High-resolution transmission electron microscopy (HR-TEM) imaging under atmospheric pressure is one of the challenging issues in biological and material sciences. Environmental TEM (ETEM) enables one to observe specimens in various gas conditions with wide ranges of pressure and temperature. Now the gas-capsulated cell with a pair of electron-transparent thin films (E-cell) is extensively used for this purpose [1], stimulated by the progress of micro electrical and mechanical system (MEMS) technology.

We propose a novel method of evaluating the high-resolution imaging in ETEM, based on contrast transfer functions (CTFs) and unscattered fraction of electrons (UFE). In this model, the effect of the electron-gas interaction in E-cell and the electron-solid interaction in electron-transparent films are treated as scattering objects for incident and transmitted electrons, leading to the image deterioration. They are separated into two parts, pre-specimen scattering object above the specimen, and postspecimen scattering object below it. The former object induces energy and angular spreads, thus the CTF of the microscope is modified. The latter object breaks the phase information of the transmitted electrons, and only the UFE carries the phase contrast amplitude.

In order to make a comparison with experiments, we employed a newly developed E-cell [2], installed in $300 \mathrm{keV}$ TEM H-9500. It is equipped with thin $(<30 \mathrm{~nm}) \mathrm{Si}_{3} \mathrm{~N}_{4}$ amorphous membranes and gas flow tubes. The distance between two membranes is $1.0 \mathrm{~mm}$. The specimen is placed on the heating element in the middle of the cell. The air $\left(\mathrm{N}_{2}: \mathrm{O}_{2}=4: 1\right)$ is used as a gas in the cell, and the pressure is measured by a MEMS pressure gage. Figure 1 shows typical ETEM images of a $\mathrm{Si}$ particle. The lattice fringe of $0.314 \mathrm{~nm}$ is clearly observed in the condition of $1 \times 10^{4} \mathrm{~Pa}(0.1 \mathrm{~atm})$ and $600^{\circ} \mathrm{C}$.

Figures 2(a) and 2(b) show the calculated CTFs of the microscope used, which are modified after passing through the pre-specimen scattering object. In Fig. 2(a) the effect of the 30-nm-thick $\mathrm{Si}_{3} \mathrm{~N}_{4}$ membrane is shown. The CTFs modified by the angular and energy spreads are shown by the (red) solid and (blue) dashed curves, respectively. The (black) dotted curve represents the original CTF without E-cell. The CTFs modified by the $0.5-\mathrm{mm}$ air space of $1 \times 10^{4} \mathrm{~Pa}$ are presented in Fig. 2(b) in the same manner. In both cases the modification of the CTF is minor, and a reasonable amount of the amplitude still remains, supporting the experimentally observed lattice image shown in Fig. 1(b). Figures 2(c) and 2(d) show the calculated UFEs for $\mathrm{Si}_{3} \mathrm{~N}_{4}$ membrane and air, respectively. The threshold above which the lattice imaging becomes possible is not obvious. In the present case (i.e., 30 -nm-thick $\mathrm{Si}_{3} \mathrm{~N}_{4}$ and $0.5-\mathrm{mm}$ air space of $1 \times 10^{4} \mathrm{~Pa}$ ), however, the total UFE yields $33 \%$, giving one of the reasonable criteria of UFE for high-resolution imaging in ETEM.

References

[1] e.g., T. Kamino et al., J. Electron Microsc. 54 (2005) 497-503.

[2] T. Yaguchi et al., Microsc. Microanal. 16 (Suppl 2) (2010) 302. 


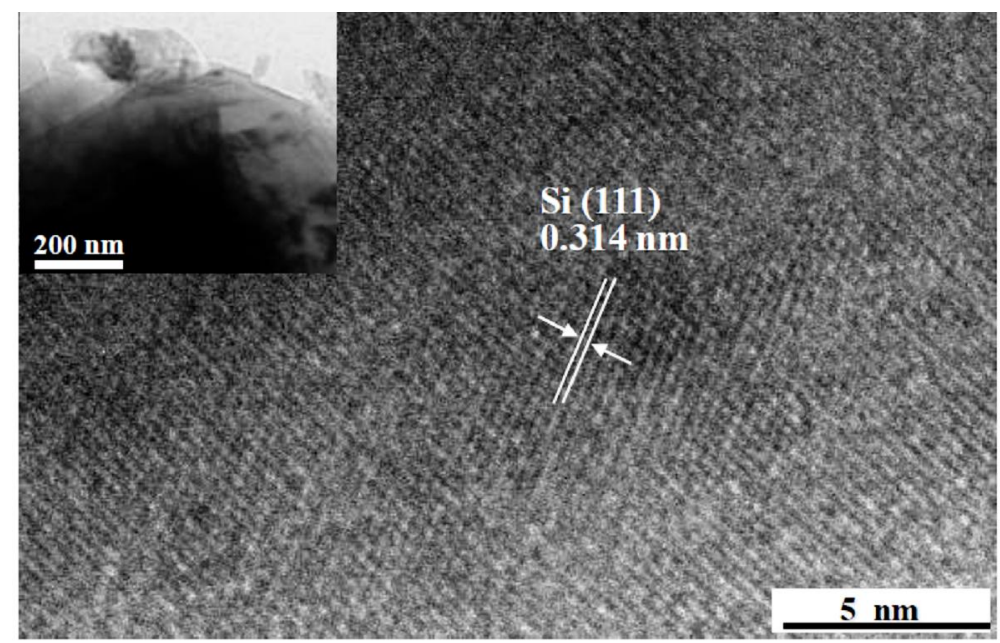

FIG. 1. HR-TEM imaging of a crystalline silicon particle observed at $600^{\circ} \mathrm{C}$ in the air pressure of $1 \times 10^{4}$ $\mathrm{Pa}$ with thin $(<30 \mathrm{~nm}) \mathrm{Si}_{3} \mathrm{~N}_{4}$ windows $1.0 \mathrm{~mm}$ apart. Inset: Low magnification image.
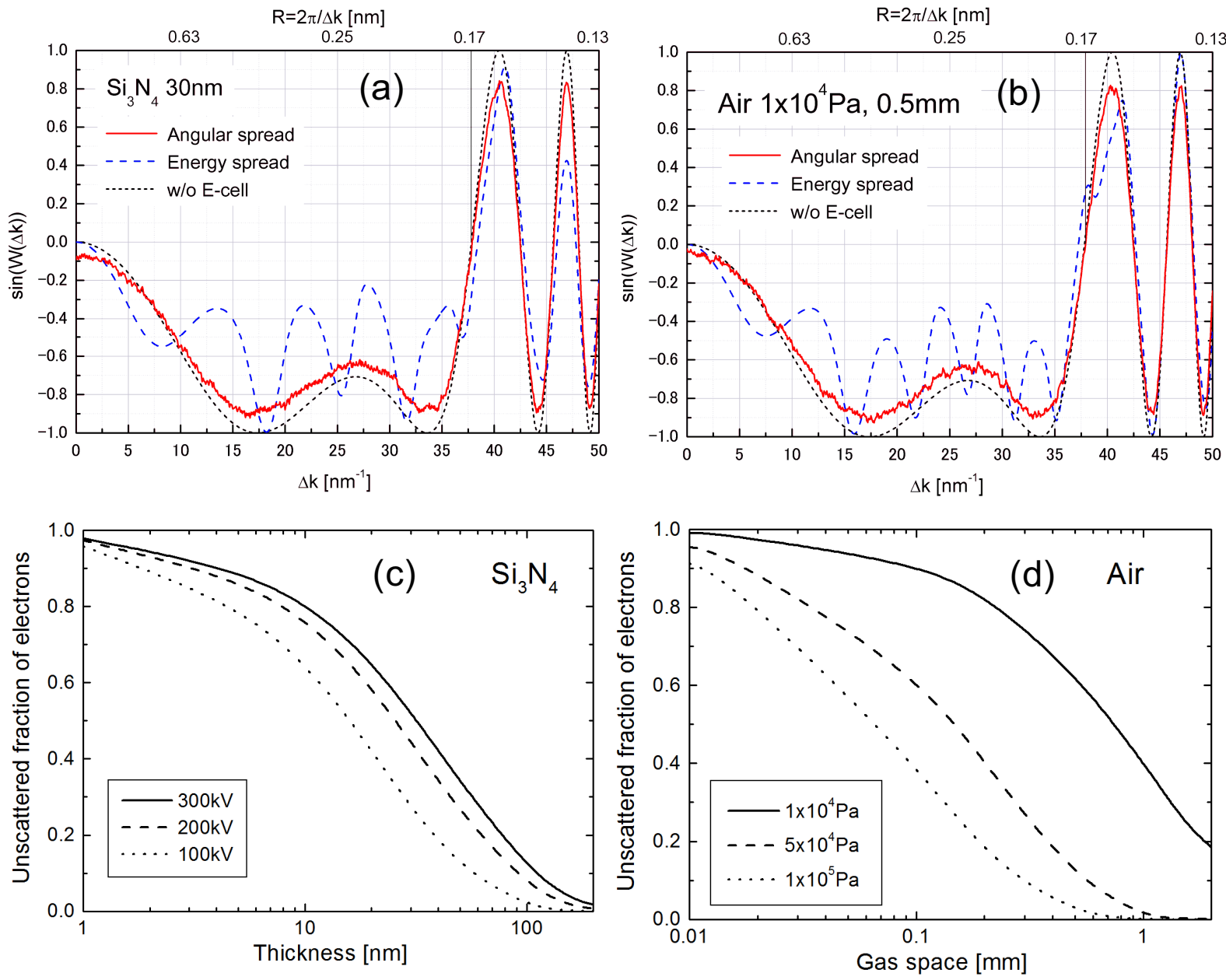

FIG. 2. (a) Contrast transfer function (CTF) modified by the interaction with a 30 -nm-thick $\mathrm{Si}_{3} \mathrm{~N}_{4}$ window, (b) CTF modified by the interaction with $0.5-\mathrm{mm}$ air space of $1 \times 10^{4} \mathrm{~Pa}$, (c) Unscattered fraction of electrons (UFE) for $\mathrm{Si}_{3} \mathrm{~N}_{4}$ membrane, (d) UFE for air. 